\title{
Editorial
}

\section{Advanced Information Technology Convergence 2017}

\author{
Jucheng Yang, ${ }^{1}$ Anthony T. S. Ho, ${ }^{2}$ Hui Cheng, ${ }^{3}$ Sook Yoon, ${ }^{4}$ and Lu Liu ${ }^{5}$ \\ ${ }^{1}$ College of Computer Science and Information Engineering, Tianjin University of Science and Technology, Tianjin, China \\ ${ }^{2}$ Department of Computing, School of Electronics and Physical Sciences, University of Surrey, Guildford, UK \\ ${ }^{3}$ School of Computing and Mathematical Sciences, Liverpool John Moores University, Liverpool, UK \\ ${ }^{4}$ Department of Computer Engineering, Mokpo National University, Jeonnam, Republic of Korea \\ ${ }^{5}$ College of Engineering and Technology, University of Derby, Derby, UK \\ Correspondence should be addressed to Jucheng Yang; jcyang@tust.edu.cn
}

Received 3 May 2017; Accepted 4 May 2017; Published 29 May 2017

Copyright (C) 2017 Jucheng Yang et al. This is an open access article distributed under the Creative Commons Attribution License, which permits unrestricted use, distribution, and reproduction in any medium, provided the original work is properly cited.

Nowadays, the tremendous growth and usage of information technologies led to rapid development in the various aspects of advances in convergence and hybrid information technology, such as IT convergence in signal and image processing, human-computer interaction, robotics, transportation system, and health care. The spread of current technologies is more emanative at all stages of research, development, diffusion, and use. Moreover, different regions of research and applications are often integrated together to achieve better performance, solve problems, and restructure systems, as well as improve the computational intelligence in both theoretical and practical aspects. Hence, the convergence of information technologies could lead to the new stage of innovation with significant increasing speed.

This special issue in 2017 is aimed to provide state-ofthe-art publication of refereed, high quality original research papers in all branches of the convergence technologies and its applications, such as signal and image processing for IT convergence, web and database technology for IT convergence, IT convergence in health care, robotics, transportation system, and Big Data technology. It provides a platform for researchers to comprehensively share ideas, problems, and solutions related to advanced information technology convergence with various topics.

The special issue consists of 8 papers whose brief summaries are listed below.

"The Channel Compressive Sensing Estimation for Power Line Based on OMP Algorithm" by Y. Zhang et al. analyzes the transmission characteristics of the power line channel and model it with mathematics channel. A new method about the power line channel estimation based on compressive sensing is proposed, which can collect and extract the effective parameters of the power line channel to storage with very little space.

"Study of SAW Based on a Micro Force Sensor in Wireless Sensor Network" by J. Wang et al. investigates wireless sensor network (WSN) acquisition nodes and analyzes the relationship between the frequency and actual pressure values of sensor nodes. Also, the sensitive mechanism of the surface acoustic wave (SAW) based on a micro force sensor is researched, and the principle of least squares method is used to establish a transformation model of frequency and pressure for the SAW sensor.

"Image Encryption Algorithm Based on a Novel Improper Fractional-Order Attractor and a Wavelet Function Map" by J. Zhao et al. presents a three-dimensional autonomous chaotic system with high fraction dimension. The image encryption algorithm is proposed based on the continuous chaos and the discrete wavelet function map.

"Ferrography Wear Particles Image Recognition Based on Extreme Learning Machine" by Q. Li et al. proposes to employ extreme learning machine (ELM) for ferrography wear particles image recognition, which shows its advantages compared to the traditional feedforward neural network based on gradient training algorithms. More specifically, the shape features, color features, and texture features of five typical kinds of wear particles are extracted as the input of the ELM classifier and five types of wear particles are set as the output of the ELM classifier. 
"Online Behavior Analysis-Based Student Profile for Intelligent E-Learning" by K. Liang et al. analyzes the relation indicators of E-Learning to build the student profile and gives countermeasures. By adopting the similarity computation and Jaccard coefficient, a system model is designed to clean and dig into the educational data and the students' learning attitude and the duration of learning behavior are also used to establish student profile. Moreover, an intelligent guide model is presented to guide both E-Learning platform and learners to improve learning things according to the ELearning resources and learner behaviors.

"A Fast and Robust Key Frame Extraction Method for Video Copyright Protection" by Y. Shi et al. proposes a key frame extraction method for video copyright protection. The fast and robust method is based on frame difference with low level features, including color feature and structure feature. A two-stage method is used to extract accurate key frames to cover the content for the whole video sequence.

"Algebraic Cryptanalysis Scheme of AES-256 Using Gröbner Basis" by K. Zhao et al. proposes a zero-dimensional Gröbner basis construction method by choosing suitable term order and variable order after performing an in-depth study on the linear transformation and the system of multivariate polynomial equations of AES-256. Also, an algebraic cryptanalysis scheme of AES-256 using Gröbner basis is proposed based on the detailed construction process of the zero-dimensional Gröbner basis.

"Acoustic Log Prediction on the Basis of Kernel Extreme Learning Machine for Wells in GJH Survey, Erdos Basin" by J. Cao et al. proposes using kernel extreme learning machine (KELM) to predict missing sonic DT logs when only common logs are available. The common logs are set as predictors and the DT log is the target. By using KELM, a prediction model is firstly created based on the experimental data and then confirmed and validated by blind-testing the results in wells containing both the predictors and the target DT values used in the supervised training. Finally the optimal model is set up as a predictor.

Jucheng Yang

Anthony T. S. Ho

Hui Cheng

Sook Yoon

Lu Liu 


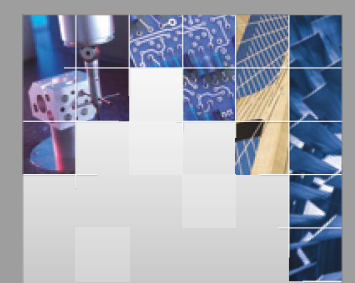

\section{Enfincering}
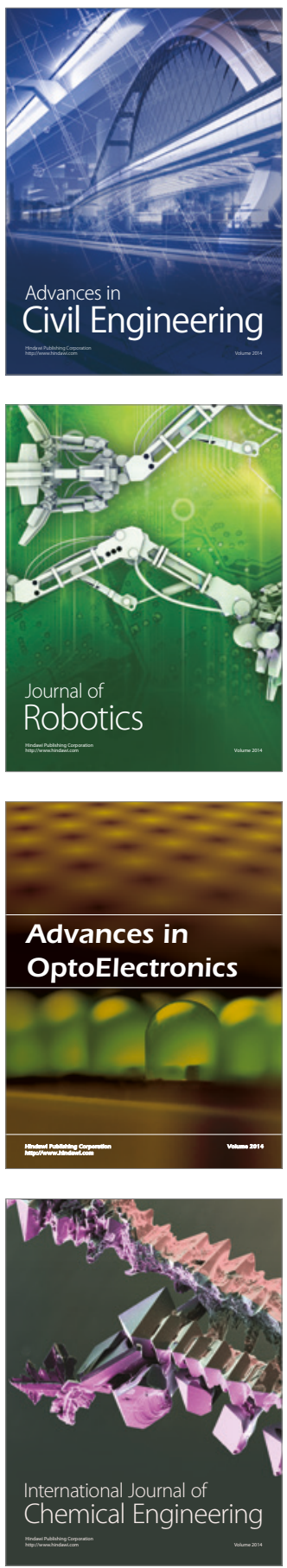

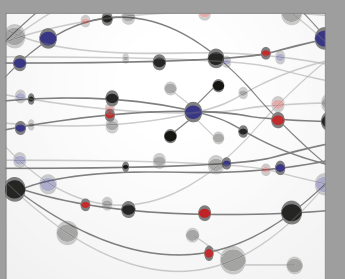

The Scientific World Journal

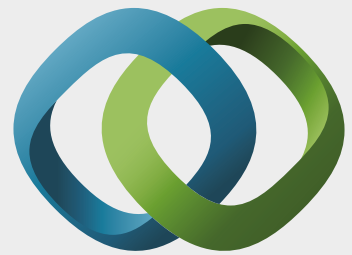

\section{Hindawi}

Submit your manuscripts at

https://www.hindawi.com
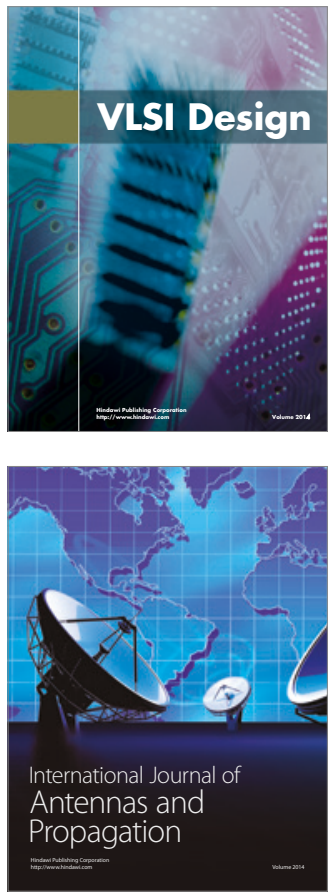

\section{Rotating}

Machinery
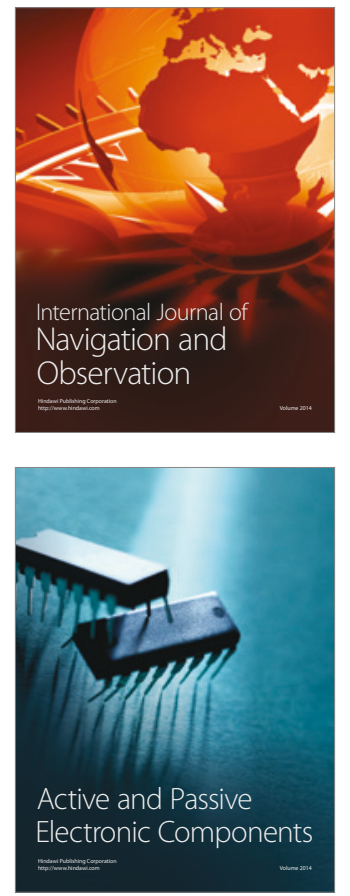
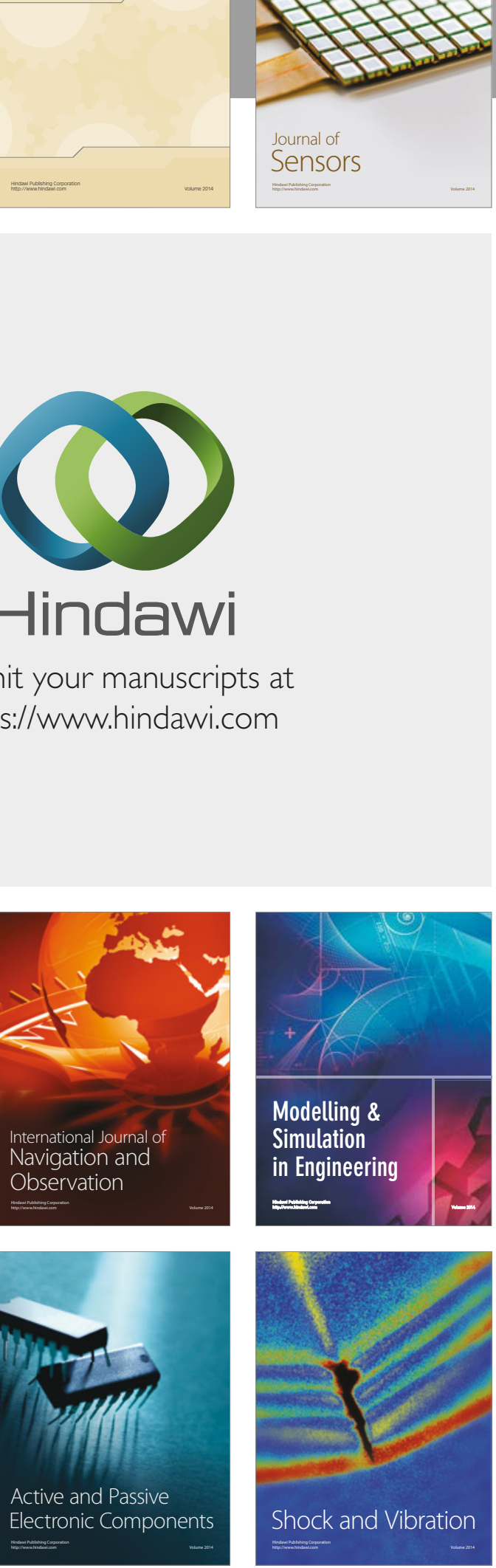
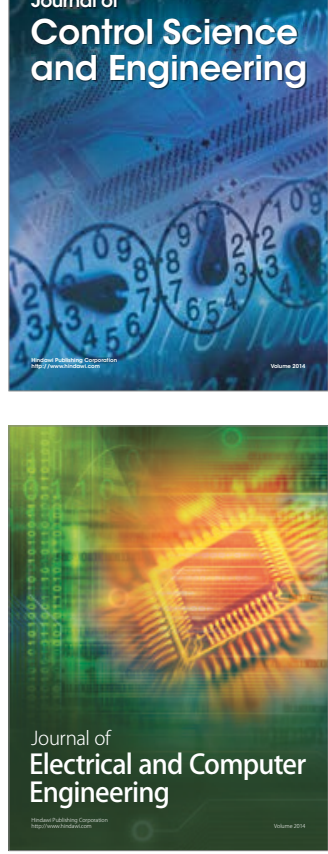

Distributed

Journal of

Control Science

and Engineering
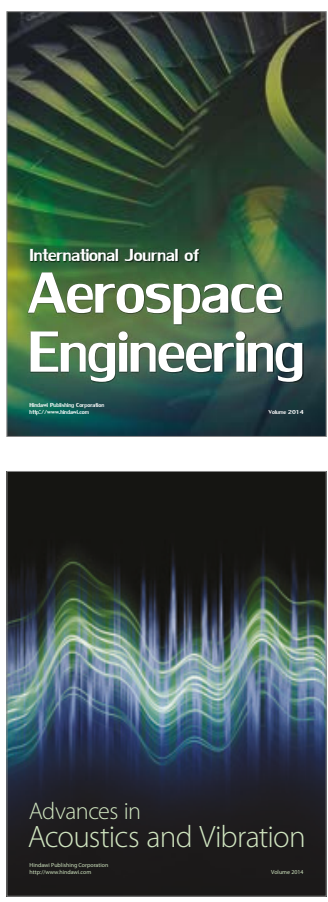

Sensor Networks 